UCRHEP-T279

June 2000

\title{
Verifiable Model of Neutrino Masses from Large Extra Dimensions
}

\author{
Ernest $\mathbf{M a}^{1}$, Martti Raidal ${ }^{1,2}$, and Utpal Sarkar ${ }^{1,3}$ \\ ${ }^{1}$ Department of Physics, University of California, Riverside, California 92521, USA \\ 2 National Institute of Chemical and Biological Physics, Rävala 10, 10143, Estonia \\ ${ }^{3}$ Physical Research Laboratory, Ahmedabad 380 009, India
}

\begin{abstract}
We propose a new scenario of neutrino masses with a Higgs triplet $\left(\xi^{++}, \xi^{+}, \xi^{0}\right)$ in a theory of large extra dimensions. Lepton number violation in a distant brane acts as the source of a very small trilinear coupling of $\xi$ to the standard Higgs doublet in our brane. Small realistic Majorana neutrino masses are naturally obtained with the fundamental scale $M_{*} \sim \mathcal{O}(1) \mathrm{TeV}$, foretelling the possible discovery of $\xi\left(m_{\xi} \lesssim M_{*}\right)$ at future colliders. Decays of $\xi^{++}$into same-sign dileptons are fixed by the neutrino mass matrix. Observation of $\mu-e$ conversion in nuclei is predicted.
\end{abstract}


Recently it has been proposed [1] that the fundamental scale of quantum gravity could be as low as a few $\mathrm{TeV}$. In this theory, our four-dimensional world (called a 3-brane) is localized to only one point in a space having $n$ extra dimensions (the bulk). Assuming that gravity is the only field which propagates in these extra dimensions, the fact that it is so weak in our world may be understood. Since there are no scales larger than a few $\mathrm{TeV}$ in this theory, the smallness of neutrino masses requires a different explanation than the usual one that it is inversely proportional to some very large mass. There are already proposals in this direction, where singlet fermions are introduced in the bulk [2, 3]. They become the right-handed partners of the observed left-handed neutrinos, but with small Dirac masses - the result of being suppressed by the volume of the extra dimensions. Note first that this mechanism by itself does not generate a Majorana mass. Note also that such a model of neutrino masses, like any other before it, has no distinct experimental signatures which would set it apart from any other. Furthermore, the existence of singlet fermions in the bulk is strongly constrained by supernova data to have an upper bound of $210 / \sqrt{n}$ TeV on the fundamental scale involved [4].

In this Letter we propose a simple, verifiable scenario in which the distant breaking [5] of lepton number is used to generate small Majorana neutrino masses through a scalar Higgs triplet [6] localized in our brane. The trilinear interaction of the triplet with the standard Higgs doublet is induced by the "shining" of a scalar singlet which communicates the violation of lepton number from a distant brane to our world through the large extra space dimensions. Thus the amount of lepton number violation in our world is suppressed by the distance between us and the other brane. Because the Higgs triplet should not be heavier than the fundamental scale of the extra dimensions, this scenario provides the basis for a rich and robust phenomenology of new observable processes at future high-energy colliders as well as low-energy precision experiments. 
The Higgs triplet may be produced at the upgraded Tevatron at Fermilab, the Large Hadron Collider (LHC) at CERN, and perhaps a future linear collider (LC) or muon collider (MC) or both. The decay branching fractions of the doubly charged member $\xi^{++}$of the Higgs triplet into same-sign dileptons or the cross sections of the resonant processes $e^{-} e^{-}\left(\mu^{-} \mu^{-}\right) \rightarrow l_{i}^{-} l_{j}^{-}$may then be used to determine the magnitude of each element of the neutrino mass matrix completely and uniquely up to an overall scale factor. Whereas lepton-number violating processes are suppressed at low energies (because their amplitudes are proportional to the neutrino mass matrix), large lepton-flavor violation through the Higgs triplet is possible. Based on current neutrino-oscillation data, the rate of coherent $\mu-e$ conversion in nuclei, with an amplitude enhanced by the large factor $\ln \left(m_{\xi^{++}}^{2} / m_{\mu}^{2}\right)$ over that of $\mu \rightarrow e \gamma$, is predicted to be observable at the proposed Muon Electron Conversion (MECO) experiment [7] at Brookhaven National Laboratory (BNL).

We extend the minimal standard model of particle interactions to include a heavy Higgs triplet $\left(\xi^{++}, \xi^{+}, \xi^{0}\right)$ with the interaction

$$
\mathcal{L}=f_{i j}\left[\xi^{0} \nu_{i} \nu_{j}+\xi^{+} \frac{\left(\nu_{i} l_{j}+l_{i} \nu_{j}\right)}{\sqrt{2}}+\xi^{++} l_{i} l_{j}\right]+h . c .
$$

implying that $\xi$ carries lepton number $L=-2$. The resulting Majorana neutrino mass matrix is then given by

$$
\left(\mathcal{M}_{\nu}\right)_{i j}=2 f_{i j}\left\langle\xi^{0}\right\rangle
$$

where $\left\langle\xi^{0}\right\rangle$ is the vacuum expectation value (VEV) of $\xi^{0}$, hence lepton number must be violated somehow. This may be achieved with the following addition to the Lagrangian:

$$
\mathcal{L}^{\prime}=h \chi\left(\bar{\xi}^{0} \phi^{0} \phi^{0}-\sqrt{2} \xi^{-} \phi^{+} \phi^{0}+\xi^{--} \phi^{+} \phi^{+}\right)+h . c .,
$$

where $\chi$ is a complex neutral scalar singlet with $L=-2$. We then obtain [6]

$$
\left\langle\xi^{0}\right\rangle \simeq \frac{h\langle\chi\rangle\left\langle\phi^{0}\right\rangle^{2}}{m_{\xi}^{2}} .
$$


In other words, the breaking of $L$ by $\langle\chi\rangle$ is communicated to the lepton sector through $\langle\xi\rangle$. As the neutrino masses are very small, we need $m_{\xi}$ to be very large in Eq. (4). Suppose $m_{\xi}$ is only of order $1 \mathrm{TeV}$, then $h\langle\chi\rangle$ would have to be very small, i.e. $h\langle\chi\rangle \sim 33 \mathrm{eV}$, if $\left\langle\xi^{0}\right\rangle \sim 1 \mathrm{eV}$, using $\left\langle\phi^{0}\right\rangle=174 \mathrm{GeV}$. However, $\chi$ is a singlet, and as such, its VEV is expected to be large, so this requires what we normally would consider as extremely unnatural fine tuning. However, in a theory of large extra dimensions, a small $\langle\chi\rangle$ may arise naturally, thus allowing $m_{\xi}$ to be of order $1 \mathrm{TeV}$ and be directly observed experimentally.

The standard model fields together with $\xi$ are localized in our world (a 3-brane $\mathcal{P}$ at $y=0$ ) and are blind to the extra space dimensions. Lepton number is assumed to be conserved as far as these fields are concerned. The singlet $\chi$ is special; it propagates also in the bulk carrying lepton number $L=-2$ and interacts in our brane according to Eq. (3). The violation of lepton number happens at a distant 3-brane which then gets communicated to our brane through $\langle\chi\rangle$.

We assume the existence of a field $\eta$ which is localized in a distant 3-brane $\left(\mathcal{P}^{\prime}\right)$ situated at a point $y=y_{*}$ in the extra dimensions. It is a singlet under the standard model but has $L=2$ and couples to $\chi$ (with $L=-2$ ). When the field $\eta$ acquires a VEV, lepton number is broken maximally in the other brane. It will then act as a point source for $L$ violation, and the field $\chi$ is the messenger which carries it to our wall (the interface between our brane and the bulk). The "shining" of $\chi$ at all points in our world is the mechanism [5] which breaks lepton number and gives mass to the neutrinos.

At energies much below the fundamental scale $M_{*}$, the lepton-number violating effect will be suppressed by the distance between the source brane at $\mathcal{P}^{\prime}$ and our brane at $\mathcal{P}$. We assume that the source brane is situated at the farthest point in the extra dimensions so that $\left|y_{*}\right|=r$ is the radius of compactification and it is related to the fundamental scale $M_{*}$ 
and the reduced Planck scale $\left(M_{P}=2.4 \times 10^{18} \mathrm{GeV}\right)$ by the relation

$$
r^{n} M_{*}^{n+2} \sim M_{P}^{2}
$$

This explains why lepton number is only violated weakly in our world.

We assume here that the source brane has the same dimensional structure as our world and there are $n$ extra dimensions. In our world $(\mathcal{P})$ the field $\chi$ has only the lepton-number conserving interaction

$$
\mathcal{S}_{\chi}=h \int_{\mathcal{P}} d^{4} x \xi^{\dagger}(x) \phi(x) \phi(x) \chi(x, y=0)
$$

In the other brane $\left(\mathcal{P}^{\prime}\right)$ the field $\chi$ couples to the field $\eta$ through the interaction

$$
\mathcal{S}_{\text {other }}=\int_{\mathcal{P}^{\prime}} d^{4} x^{\prime} \mu^{2} \eta\left(x^{\prime}\right) \chi\left(x^{\prime}, y=y_{*}\right)
$$

where $\mu$ is a mass parameter. Lepton number violation from $\langle\eta\rangle$ is carried by $\chi$ to our world through its "shined" value $\langle\chi\rangle$ :

$$
\langle\chi(x, y=0)\rangle=\Delta_{n}(r)\left\langle\eta\left(x, y=y_{*}\right)\right\rangle
$$

where $\langle\eta\rangle$ acts as a point source, and $\Delta_{n}(r)$ is the Yukawa potential in $n$ transverse dimensions, i.e. [5]

$$
\Delta_{n}(r)=\frac{1}{(2 \pi)^{\frac{n}{2}} M_{*}^{n-2}}\left(\frac{m_{\chi}}{r}\right)^{\frac{n-2}{2}} K_{\frac{n-2}{2}}\left(m_{\chi} r\right),
$$

$K$ being the modified Bessel function. If the mass of the carrier field $\chi$ is large $\left(m_{\chi} r \gg 1\right)$, it has the profile

$$
\langle\chi\rangle \approx \frac{m_{\chi}^{\frac{n-3}{2}}}{2(2 \pi)^{\frac{n-1}{2}} M_{*}^{n-3}} \frac{e^{-m_{\chi} r}}{r^{\frac{n-1}{2}}} .
$$

The suppression here is exponential, hence the amount of lepton-number violation in our world is very small, but its precise value depends sensitively on $m_{\chi}$. An interesting alternative is to have a light carrier field with a mass less than $1 / r$. However, it should be larger than 
about $(0.1 \mathrm{~mm})^{-1}$, to be consistent with the present experimental data on gravitational interactions.

If $m_{\chi} r \ll 1, \Delta_{n}(r)$ is logarithmic for $n=2$ and $\langle\chi\rangle$ is not suppressed. For $n>2$, the asymptotic form of the profile of $\chi$ is

$$
\langle\chi\rangle \approx \frac{\Gamma\left(\frac{n-2}{2}\right)}{4 \pi^{\frac{n}{2}}} \frac{M_{*}}{\left(M_{*} r\right)^{n-2}},
$$

which is suitably small for large $r$. Using Eqs. (2), (4), and (5), we then obtain

$$
\left(\mathcal{M}_{\nu}\right)_{i j} \approx \frac{\Gamma\left(\frac{n-2}{2}\right)}{2 \pi^{\frac{n}{2}}} h f_{i j} \frac{\langle\phi\rangle^{2} M_{*}}{m_{\xi}^{2}}\left(\frac{M_{*}}{M_{P}}\right)^{2-\frac{4}{n}} .
$$

For $n=3, M_{*} \sim 1 \mathrm{TeV}, m_{\xi} \lesssim 1 \mathrm{TeV}$ and $h \sim f_{33} \sim 0.25$, we get $\left(\mathcal{M}_{\nu}\right)_{33} \sim 0.03 \mathrm{eV}$, which is of the right magnitude for understanding atmospheric neutrino oscillations. There is a massless Goldstone boson (Majoron) corresponding to the spontaneous breaking of lepton number in this scenario. It is a singlet and its coupling to the leptons in our world is suppressed by $\langle\eta\rangle^{-1}$. It is thus completely acceptable phenomenologically.

For $n \geq 4$, this scenario requires a larger fundamental scale $M_{*}$ and is of less phenomenological interest. However, we have assumed here for simplicity the dimension of the source brane to be the same as ours, which may not be the case. The distance between the branes may also be smaller. On the other hand, such complications diminish the predictive power of the proposed mechanism; they will not be considered any further.

The only pair production mechanism of $\xi^{++}$at the LHC is the Drell-Yan process mediated by s-channel photon and Z-boson exchange [8]. Thus the production rate is enhanced by the double charge of $\xi^{++}$and is uniquely determined by the gauge couplings. To obtain the cross section of $p p \rightarrow \xi^{++} \xi^{--}$at the LHC, we have calculated the subprocesses involving the $u, \bar{u}$ and $d, \bar{d}$ collisions and convoluted them over the parton distributions given by the default set of the CERN library package PDFLIB [9]. The total cross section as a function of the triplet mass $m_{\xi^{++}}$is plotted in Fig. 11. 
Once produced, $\xi^{++}$will decay into same-sign dileptons. Because $\langle\xi\rangle$ is tiny, the decay branching fraction of $\xi^{++} \rightarrow W^{+} W^{+}$is negligible. Thus the only possible decay channels in our scenario are $\xi^{++} \rightarrow l_{i}^{+} l_{j}^{+}$with the partial rates $\Gamma_{i j}=\left|f_{i j}\right|^{2} m_{\xi} / 4 \pi$ for $i \neq j$, and $1 / 2$ smaller for $i=j$. This same-sign dilepton signal at the invariant mass of $\xi$ is very distinctive at the LHC because it is completely background-free. Assuming the total integrated luminosity of the LHC to be $1000 \mathrm{fb}^{-1}$, the reconstruction efficiency of the event to be $10 \%$ (to be very conservative) and that the predicted average of $N=-\ln (1-p)$ Poisson distributed events would provide a discovery, the cross section in Fig. 1 implies at $p=95 \%$ confidence level that $m_{\xi^{++}} \lesssim 1.2 \mathrm{TeV}$ can be probed at the LHC [300 GeV at the Tevatron]. Its decay branching fractions will then determine $\left|f_{i j}\right|$, i.e. the magnitude of each element of the neutrino mass matrix up to an overall scale factor. This is the only model of neutrino masses which has the promise of being verified from collider experiments.

Complementary measurement of $\left|f_{i j}\right|$ is provided by the resonant processes $e^{-} e^{-}\left(\mu^{-} \mu^{-}\right) \rightarrow$ $l_{i}^{-} l_{j}^{-}$at a future LC and/or MC. The $\xi^{++}$mass reach in these colliders extends up to the collision energies, which may be as high as $4 \mathrm{TeV}$. The sensitivity to $\left|f_{i j}\right|$ depends on the beam properties of the machines. The detailed estimate in Ref. [10 implies that $\left|\left(f \cdot f^{*}\right)_{i j}\right| \gtrsim 10^{-8}$ can be probed in these processes.

Consider now a phenomenological hierarchical neutrino mass matrix consistent with the atmospheric and solar neutrino results [11]:

$$
\mathcal{M}_{\nu}=m\left(\begin{array}{ccc}
a x & b & b x \\
b & x^{2} & x \\
b x & x & 1
\end{array}\right)
$$

where $m$ is the normalization mass and $0.67<x<1$ determines the $\nu_{\mu} \rightarrow \nu_{\tau}$ mixing as required by the atmospheric neutrinos. The three solutions of the solar neutrino problem correspond to (i) large-angle matter-enhanced oscillations: $a=0.006, b=0.04$; (ii) smallangle matter-enhanced oscillations: $a=4 \times 10^{-6} ; b=0.0012$; and (iii) vacuum oscillations: 
$a=0.0002, b=0.0012$.

Given the pattern of $f_{i j}$ via the neutrino mass matrix of Eq. (13), lepton-flavor violation through $\xi$ exchange may be observable at low energies. The amplitude of $\mu-e$ conversion in nuclei is enhanced by $\ln \left(m_{\xi^{++}}^{2} / m_{\mu}^{2}\right)$ compared to that of $\mu \rightarrow e \gamma$ [12]. Planned experiments will reach the sensitivity of $10^{-16}$ for $\mu-e$ conversion in aluminum [0] and $10^{-14}$ for $\mu \rightarrow e \gamma$ [13]. The matrix element of photonic conversion is given by $\mathcal{M}=\left(4 \pi \alpha / q^{2}\right) j^{\mu} J_{\mu}$, where $q$ is the momentum transfer with $q^{2} \approx-m_{\mu}^{2}, J$ is the hadronic current, and

$$
\begin{aligned}
j^{\lambda}= & \bar{u}\left(p_{e}\right)\left[\left(f_{E 0}+\gamma_{5} f_{M 0}\right) \gamma_{\nu}\left(g^{\lambda \nu}-\frac{q^{\lambda} q^{\nu}}{q^{2}}\right)\right. \\
& \left.+\left(f_{M 1}+\gamma_{5} f_{E 1}\right) i \sigma^{\lambda \nu} \frac{q_{\nu}}{m_{\mu}}\right] u\left(p_{\mu}\right)
\end{aligned}
$$

is the leptonic current. The coherent $\mu-e$ conversion ratio in nuclei is given by

$$
R_{\mu e}=\frac{8 \alpha^{5} m_{\mu}^{5} Z_{e f f}^{4} Z\left|\overline{F_{p}}\left(p_{e}\right)\right|^{2}}{\Gamma_{\text {capt }}} \frac{\xi_{0}^{2}}{q^{4}}
$$

where $\xi_{0}^{2}=\left|f_{E 0}+f_{M 1}\right|^{2}+\left|f_{E 1}+f_{M 0}\right|^{2}$, and for ${ }^{13} \mathrm{Al}, Z_{e f f}^{A l}=11.62, \bar{F}_{p}^{A l}(q)=0.66$, and $\Gamma_{c a p t}^{A l}=7.1 \times 10^{5} \mathrm{~s}^{-1}[14]$. We calculate the form factors induced by the one-loop diagrams involving $\xi^{++}$and obtain

$$
\begin{aligned}
& f_{E 0}=f_{M 0}=\sum_{l} \frac{f_{\mu l} f_{l e}^{*}}{24 \pi^{2}}\left[4 s_{l}+r F\left(s_{l}\right)\right] \\
& f_{M 1}=-f_{E 1}=\sum_{l} \frac{f_{\mu l} f_{l e}^{*}}{24 \pi^{2}} s_{\mu}
\end{aligned}
$$

where

$$
F\left(s_{l}\right)=\ln s_{l}+\left(1-\frac{2 s_{l}}{r}\right) \sqrt{1+\frac{4 s_{l}}{r}} \ln \left[\frac{\sqrt{r+4 s_{l}}+\sqrt{r}}{\sqrt{r+4 s_{l}}-\sqrt{r}}\right],
$$

and $r=-q^{2} / m_{\xi^{++}}^{2}, s_{l}=m_{l}^{2} / m_{\xi^{++}}^{2}, l=e, \mu, \tau$. In the interesting limit $s_{l} \rightarrow 0$, we get $F\left(s_{l}\right) \rightarrow \ln r$.

For numerical estimates we assume $m=0.03 \mathrm{eV}$ in Eq. (13) and the large-angle matterenhanced oscillation solution to the solar neutrino problem. In Fig. 2 we plot the ratio of 
$\mu-e$ conversion in aluminum as a function of the mass $m_{\xi^{++}}$and coupling $h$, assuming the fundamental scale to be $M_{*}=1 \mathrm{TeV}$. The behavior of this ratio can be understood from Eq. (12): a fixed neutrino mass implies $f \propto m_{\xi^{++}}^{2}$ and $f \propto 1 / h$. Notice the complementarity of collider and $\mu-e$ conversion experiments. For small $m_{\xi^{++}}, R_{\mu e}$ is suppressed while the collider cross section is kinematically enhanced, and vice versa. Thus for $M_{*}=1 \mathrm{TeV}$, the MECO experiment should see a signal unless $m_{\xi^{++}} \lesssim 300 \mathrm{GeV}$. If $m_{\xi^{++}} \sim M_{*}$, which is the most likely situation in our scenario, and $h \sim 1$ which minimizes the signal, then MECO will test our model up to the scale $M_{*} \sim 7 \mathrm{TeV}$.

In conclusion, we have proposed a verifiable scenario of large extra dimensions in which we invoke the distant breaking of lepton number to obtain small Majorana neutrino masses, using a Higgs triplet $\xi$ localized in our world. In this scenario, the fundamental scale $M_{*}$ may be a few $\mathrm{TeV}$, thus $m_{\xi} \lesssim M_{*}$ makes $\xi$ kinematically accessible at the LHC and at future lepton colliders. Our model has the unique feature that the decay branching fractions of $\xi^{++}$into charged leptons $l_{i}^{+} l_{j}^{+}$determine directly $\left|f_{i j}\right|$, where $f_{i j}$ is the neutrino mass matrix up to an overall scale factor. Using present neutrino data, we also predict observable $\mu-e$ conversion in nuclei. In particular, for hierarchical neutrino masses and the large-angle matter-enhanced solution for the solar neutrino problem, the MECO experiment can test our model up to $m_{\xi} \sim M_{*} \sim 7 \mathrm{TeV}$.

Acknowledgement. This work was supported in part by the U. S. Department of Energy under Grant No. DE-FG03-94ER40837.

\section{References}

[1] N. Arkani-Hamed, S. Dimopoulos, and G. Dvali, Phys. Lett. B429, 263 (1998); I. Antoniadis, N. Arkani-Hamed, S. Dimopoulos, and G. Dvali, Phys. Lett. B436, 257 
(1998); N. Arkani-Hamed, S. Dimopoulos, and G. Dvali, Phys. Rev. D59, 086004 (1999).

[2] N. Arkani-Hamed, S. Dimopoulos, G. Dvali, and J. March-Russell, hep-ph/9811448; K. R. Dienes, E. Dudas, and T. Gherghetta, Nucl. Phys. B557, 25 (1999).

[3] A. E. Faraggi and M. Pospelov, Phys. Lett. B458, 237 (1999); A. Das and O. C. W. Kong, Phys. Lett. B470, 149 (1999); R. N. Mohapatra, S. Nandi, and A. PerezLorenzana, Phys. Lett. B466, 115 (1999); G. Dvali and A. Yu. Smirnov, Nucl. Phys. B563, 63 (1999); R. Barbieri, P. Creminelli, and A. Strumia, hep-ph/0002199.

[4] L. N. Chang, O. Lebedev, W. Loinaz, and T. Takeuchi, hep-ph/0005236.

[5] N. Arkani-Hamed and S. Dimopoulos, hep-ph/9811353; N. Arkani-Hamed, L. Hall, D. Smith, and N. Weiner, Phys. Rev. D61, 116003 (2000); Y. Sakamura, hep-ph/9912511.

[6] E. Ma and U. Sarkar, Phys. Rev. Lett. 80, 5716 (1998).

[7] M. Bachmann et al., MECO Collaboration, Research Proposal E940 at BNL (1997).

[8] K. Huitu, J. Maalampi, A. Pietila, and M. Raidal, Nucl. Phys. B487, 27 (1997).

[9] H. Plothow-Besch, Comp. Phys. Comm. 75, 396 (1993).

[10] F. Cuypers and M. Raidal, Nucl. Phys. B501, 3 (1997); M. Raidal, Phys. Rev. D57, 2013 (1998).

[11] Y. Fukuda et al., Super-Kamiokande Collaboration, Phys. Rev. Lett. 81, 1158 (1998); Phys. Rev. Lett. 81, 1562 (1998); Phys. Lett. B433, 9 (1998).

[12] M. Raidal and A. Santamaria, Phys. Lett. B421, 250 (1998).

[13] L. M. Barkov et al., Research Proposal at PSI (1999).

[14] H. C. Chiang et al., Nucl. Phys. A559, 526 (1993). 


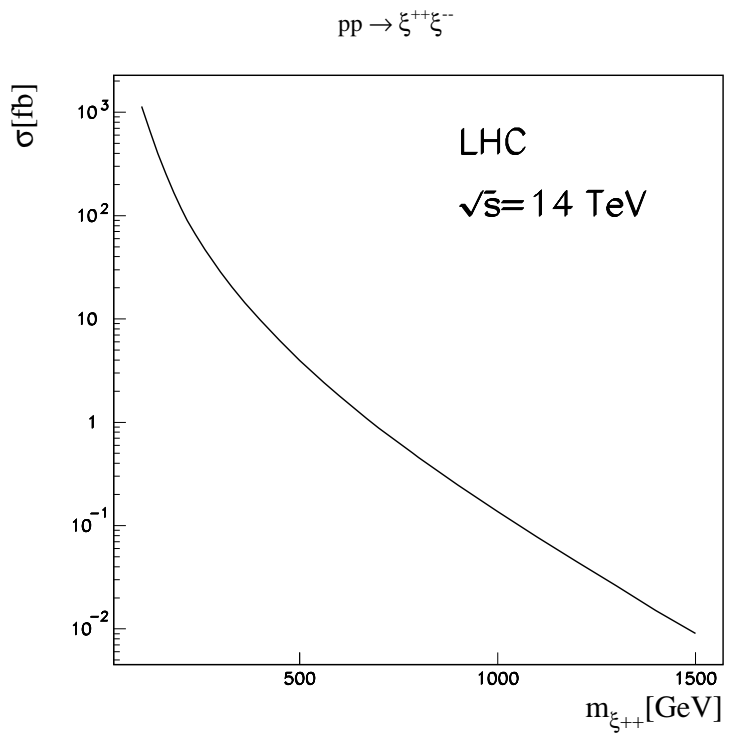

Figure 1: Cross section of $\xi^{++} \xi^{--}$Drell-Yan pair production at LHC.

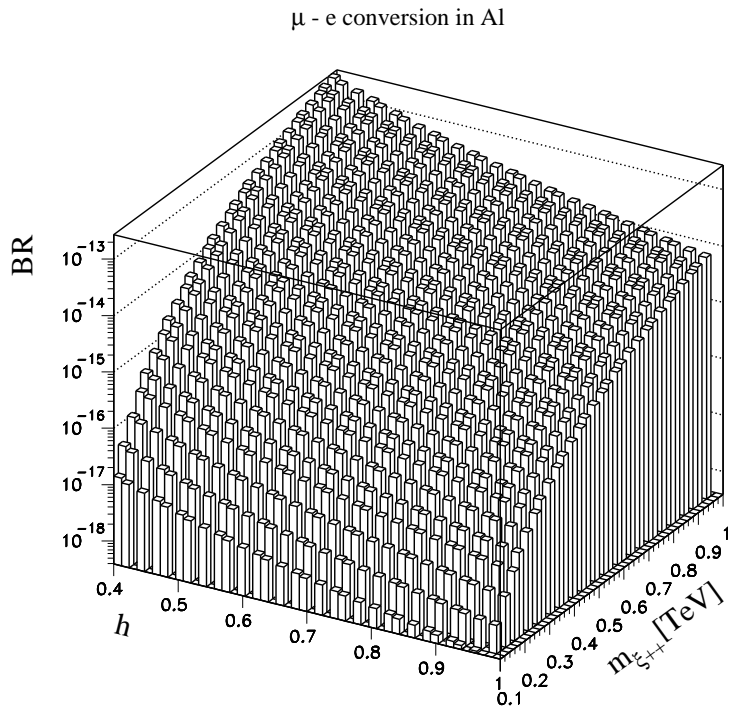

Figure 2: Rate of $\mu-e$ conversion in ${ }^{13} \mathrm{Al}$ against the $\xi^{++}$mass and Higgs self coupling $h$ for $M_{*}=1 \mathrm{TeV}$, assuming large-angle matter-enhanced solution to the solar neutrino problem. 